\title{
Carcinoma papilar en quiste tirogloso: Reporte de 4 casos y revisión de la literatura
}

\section{Papillary carcinoma in a thyroglossal duct cyst: Report of 4 cases and review of literature}

\author{
Ricardo Alarcón $\mathbf{G}^{1,2}$, Thomas Schmidt $\mathrm{P}^{1,2}$, Pablo Ortega $\mathbf{R}^{1}$, Carolina Delgado $\mathbf{S}^{3}$, \\ Fernando Casanueva $\mathrm{L}^{1}$, Matías Novoa $\mathrm{E}^{4}$.
}

\begin{abstract}
RESUMEN
Introducción: El quiste tirogloso es una de las masas cervicales más frecuentes. En $1 \%$ puede desarrollar un cáncer, siendo el más frecuente el carcinoma papilar tiroideo.

objetivo: Describir la presentación clínica, el diagnóstico, el manejo y la evolución de los casos de carcinoma papilar tiroideo en quiste tirogloso.

Material y método: Se revisaron los archivos del Departamento de Patología de la Universidad de Concepción desde 2000 a 2010 analizando las fichas clínicas y el informe de la biopsia.

Resultados: Se encontraron 4 casos de carcinoma papilar en quiste tirogloso, todos de sexo femenino con un promedio de edad de 42 años. Todos fueron sometidos a la operación de Sistrunk. Dos casos fueron sometidos a tiroidectomía total y terapia con radioyodo complementaria. Sólo un paciente presentaba un cáncer sincrónico en la tiroides. El seguimiento promedio fue de 4,7 años y no se presentaron recidivas.

Conclusiones: Se encontraron 4 casos, se analiza su presentación, diagnóstico, tratamiento y evolución.

Palabras clave: Carcinoma, papilar, tirogloso.
\end{abstract}

\section{ABSTRACT}

Introduction: Thyroglossal duct cyst is one the most frequent cervical masses. Cancer may develop from 1\% among them, thyroid papillary carcinoma being the most frequent.

Aim: To describe the clinical presentation, diagnosis, management and evolution of thyroid papillary carcinoma cases in thyroglossal duct cyst.

Material and method: A review was made of the files from the department of pathology of the Universidad de Concepción, since 2000 to 2010, analyzing clinical records and biopsy reports.

\footnotetext{
${ }^{1}$ Servicio de Otorrinolaringología, Hospital Guillermo Grant Benavente.

2Departamento de Especialidades, Facultad de Medicina, Universidad de Concepción.

${ }^{3}$ Departamento de Patología, Facultad de Medicina, Universidad de Concepción.

${ }^{4}$ Interno Medicina, Universidad de Concepción.
} 
Results: Four cases of papillary carcinoma in a thyroglossal duct cyst were found, all female with a mean of age of 42 years. All of them underwent Sistrunk procedure. Two cases underwent total thyroidectomy and complementary radioiodine therapy. Only one patient presented synchronic thyroideal cancer. The mean time of follow up consisted of 4,7 years, with no reported recurrence.

Conclusions: We found 4 cases, his presentation, diagnosis, treatment and evolution was analyzed.

Key words: Carcinoma, Papillary, Thyroglossal.

\section{INTRODUCCIÓN}

La glándula tiroides se desarrolla desde una invaginación endodérmica de la línea media entre la tercera y cuarta semana embrionaria. Esta invaginación epitelial desciende desde el agujero ciego de la lengua a través de la línea media hasta la cara anterior de los primeros anillos traqueales. El tracto epitelial derivado de este descenso se oblitera aproximadamente entre la novena y décima semana. La falla en la obliteración de este conducto genera la persistencia del conducto tirogloso y a la presencia de quistes del conducto tirogloso (QT). En forma ocasional el QT puede presentar enfermedad neoplásica. En su histología el QT presenta folículos tiroideos y epitelio escamoso de manera que las principales malignidades son los carcinomas tiroideos, especialmente el carcinoma papilar (CP) $(80 \%)$ seguido del carcinoma tiroideo mixto papilar/folicular (8\%) y el carcinoma escamoso (6\%). El otro $6 \%$ se reparte entre carcinoma de células de Hurtle, carcinoma folicular y carcinoma anaplásico ${ }^{1,2}$.

Se presentan a continuación 4 casos registrados en el Hospital Regional de Concepción en un periodo de 10 años y una revisión de la literatura, centrando la discusión en los aspectos actualmente más controvertidos del manejo.

\section{OBJETIVO}

Nuestro objetivo es describir la presentación clínica, el diagnóstico, el manejo y la evolución de los casos de CP en QT registrados en el Departamento de Patología del Hospital Regional de Concepción en un periodo de 10 años.

\section{MATERIAL Y MÉTODO}

Estudio descriptivo retrospectivo. Se revisaron los archivos del Departamento de Anatomía Patológica del Hospital Regional de Concepción desde el 1 enero del año 2000 hasta 31 de diciembre de 2010, buscando los casos de cáncer en QT. Se analizaron los informes de las biopsias registrando datos del tipo histológico, tamaño tumoral, compromiso capsular y de estructuras adyacentes. Además se analizaron las fichas clínicas obteniendo datos del paciente, estudio clínico, manejo y la evolución postratamiento.

\section{RESULTADOS}

En los 10 años revisados se diagnosticaron 4 casos de cáncer en QT. La edad promedio fue 42 años y todos fueron de sexo femenino. Todos los pacientes consultaron por misma historia clínica, masa cervical anterior, indolora, al examen físico móvil, ascendía con la deglución y la protrusión de la lengua, sin signos inflamatorios agudos ni compromiso de la piel, sin otra sintomatología asociada. A la imagenología sólo un paciente presentaba nódulos en la tiroides y ninguno adenopatías cervicales. Los datos clínicos y del estudio se presentan en la Tabla 1. La tomografía computarizada (TC) del caso 4 se presenta en la Figura 1. En dos pacientes se realizaron ecotomografía cervical y en sus informes no se describen calcificaciones intraquísticas.

Todos los pacientes fueron sometidos a una operación de Sistrunk. El tipo histológico en todos los casos fue el CP. El tamaño promedio del tumor fue $1,5 \mathrm{~cm}$. Los detalles del informe de la biopsia se presentan en la Tabla 2. La pieza quirúrgica del caso 
Tabla 1. Datos clínicos de los pacientes y del estudio preoperatorio

\begin{tabular}{|llllllll|}
\hline Caso & Motivo consulta & $\begin{array}{l}\text { Edad } \\
\text { años }\end{array}$ & Sexo & $\begin{array}{l}\text { Tamaño } \\
\text { quiste }\end{array}$ & Estudio imagen & $\begin{array}{l}\text { Adenopatías } \\
\text { cervicales }\end{array}$ & $\begin{array}{l}\text { Nódulos } \\
\text { tiroideos }\end{array}$ \\
\hline No1 & Masa cervical & 27 & Femenino & $3 \times 3 \mathrm{~cm}$ & Eco cervical & Negativo & Negativo \\
No2 & Masa cervical & 41 & Femenino & $4,5 \times 1,5 \mathrm{~cm}$ & TAC cervical & Negativo & Negativo \\
No3 & Masa cervical & 55 & Femenino & $3 \times 1,9 \mathrm{~cm}$ & TAC cervical & Negativo & Dos \\
No4 & Masa cervical & 45 & Femenino & $4 \times 2 \mathrm{~cm}$ & TAC cervical & Negativo & Negativo \\
\hline
\end{tabular}

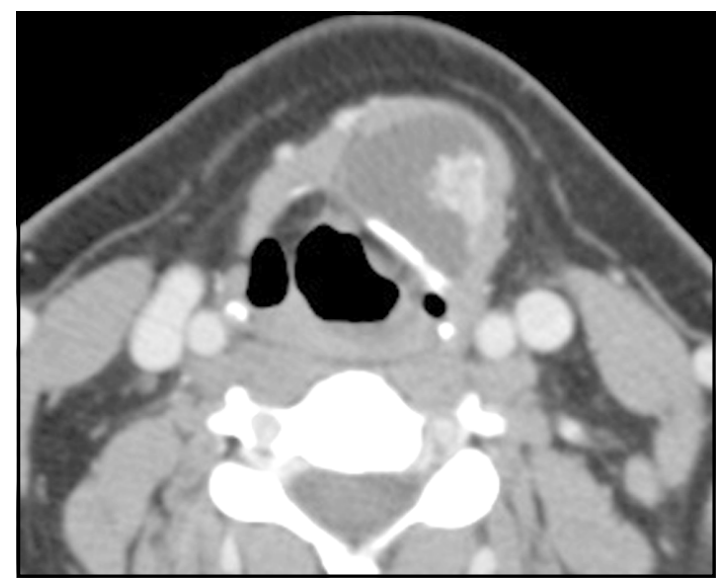

Figura 1. TC cuello caso 4

4 se presenta en la Figura 2. En relación al manejo en las pacientes 1 y 3 se asoció tiroidectomía total y terapia con radioyodo complementaria. Sólo el caso 3 presentó una neoplasia tiroidea en la pieza de tiroidectomía y correspondió a carcinoma papilar y carcinoma folicular. En el resto de las pacientes se restringió el manejo a la operación de Sistrunk. El seguimiento promedio fue de 4,75 años y ningún caso ha presentado recidiva ni metástasis cervicales 0 a distancia (Tabla 3 ).

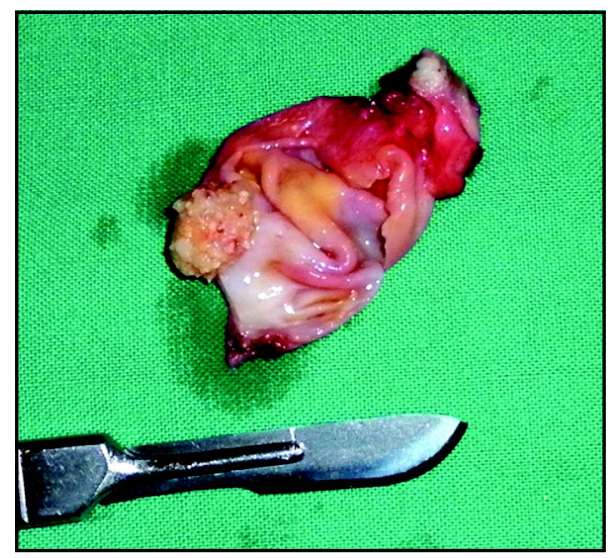

Figura 2. Pieza quirúrgica caso 4. 
Tabla 2. Hallazgos histopatológicos de la pieza de la operación de Sistrunk

\begin{tabular}{|c|c|c|c|c|c|c|c|}
\hline Caso & $\begin{array}{l}\text { Tamaño } \\
\text { tumoral }\end{array}$ & $\begin{array}{l}\text { Histología } \\
\text { carcinoma }\end{array}$ & $\begin{array}{l}\text { Papilas } \\
\text { macro }\end{array}$ & $\begin{array}{l}\text { Presencia } \\
\text { calcificación }\end{array}$ & $\begin{array}{l}\text { Invasión } \\
\text { parietal }\end{array}$ & PTLV & $\begin{array}{l}\text { Compromiso } \\
\text { borde }\end{array}$ \\
\hline $\mathrm{N}^{0} 1$ & $1,5 \mathrm{~cm}$ & Papilar & Positivo & Positivo & Negativo & Negativo & Negativo \\
\hline$N^{0} 2$ & $1,3 \mathrm{~cm}$ & Papilar & Positivo & Positivo & Invade & Negativo & Negativo \\
\hline №3 & $1,9 \mathrm{~cm}$ & Papilar & Negativo & Positivo & Atraviesa & Positivo & Positivo \\
\hline$N^{\circ} 4$ & $1,5 \mathrm{~cm}$ & Papilar & Positivo & Positivo & Negativo & Negativo & Negativo \\
\hline
\end{tabular}

PTLV: permeación tumoral linfovascular.

Tabla 3. Datos del manejo y de la evolución postratamiento

\begin{tabular}{|lllllll|}
\hline Caso & Cirugía QT & $\begin{array}{l}\text { Cirugía } \\
\text { tiroides }\end{array}$ & Radioyodo & $\begin{array}{l}\text { Supresión } \\
\text { hormonal }\end{array}$ & $\begin{array}{l}\text { Recidiva } \\
\text { (seguimiento) }\end{array}$ & $\begin{array}{l}\text { Metástasis } \\
\text { nodales }\end{array}$ \\
\hline No1 & Sistrunk & Tiroidectomía total & $\mathrm{Si}$ & $\mathrm{Si}$ & No (2 años) & Negativo \\
No2 & Sistrunk & No & $\mathrm{No}$ & $\mathrm{Si}$ & No (10 años) & Negativo \\
No3 & Sistrunk & Tiroidectomía total & $\mathrm{Si}$ & $\mathrm{Si}$ & No (6 años) & Negativo \\
No4 & Sistrunk & No & No & No & No (1 año) & Negativo \\
\hline
\end{tabular}

\section{DISCUSIÓN}

Brentano realizó el primer reporte de caso en 1911 de carcinoma en quiste tirogloso $0^{3}$, desde entonces se registran aproximadamente 200 casos en la literatura mundial, la mayoría como reporte de un caso. La presencia de carcinoma en QT bordea el $1 \%$. De 741 quistes tiroglosos extirpados en la Clínica Mayo el 0,7\% presentó un carcinoma. Así también LiVolsi ${ }^{4}$ encontró 6 carcinomas en 377 QT operados (1,5\%).

En la literatura nacional existen dos reportes con 2 casos cada uno ${ }^{15,16}$.

Normalmente la forma de presentación es la de un QT habitual, sin clínica que sugiera malignidad, encontrándose la enfermedad en el análisis histopatológico de la pieza operatoria. Existe debate si el carcinoma papilar en QT en presencia de una glándula tiroides normal corresponde a un carcinoma primario del quiste 0 una metástasis de un carcinoma tiroideo oculto. Pero la frecuente ausencia de un tumor en la tiroides y la presencia de tejido tiroideo en más del $50 \%$ de los QT apoyan el origen primario en el quiste.
Algunas características clínicas presentes podrían hacer sospechar malignidad en un quiste tirogloso: duro, fijo, irregular o acompañado de adenopatías cervicales ${ }^{2,5}$, pero estos signos rara vez se presentan.

Cuando existe sospecha de malignidad, la punción aspirativa con aguja fina (PAAF) puede ayudar al diagnóstico con sensibilidad cercana al 55\%. Este valor puede ser superior si la PAAF se realiza luego del vaciamiento del contenido del quiste $y$ especialmente si se realiza bajo visión ecográfica ${ }^{6}$. Pero su valor diagnóstico en las diferentes series es limitado.

Con respecto al estudio imagenológico hay una pequeña serie de 17 pacientes que posee una TC preoperatoria, siendo los hallazgos más frecuentes nódulo sólido dentro del quiste, calcificación, margen irregular y pared gruesa ${ }^{7,8}$. Permitiendo muchas veces con estos hallazgos sospechar una malignidad en el preoperatorio.

Uno de los aspectos controversiales en la literatura sobre el manejo de esta patología es la necesidad o no de tratamientos complementarios. Se postulan supresión hormonal, terapia con 
radioyodo, tiroidectomía, y el manejo de las metástasis nodales con vaciamiento ganglionar cervical.

El manejo del QT incluye la resección del quiste, del trayecto, y del cuerpo del hioides, procedimiento descrito por Sistrunk en 1920. Existe una diferencia significativa con respecto a la recidiva y a la sobrevida entre la simple extirpación del quiste y la realización de la operación de Sistrunk $k^{1,2,4,5}$.

La incidencia de carcinoma tiroideo sincrónico (en tiroides ecográficamente normal) es del orden del $30 \%$, porcentaje comparable a prevalencia de carcinoma tiroideo incidental en autopsias que dependiendo de la población estudiada y el método utilizado se encuentra cercano al $35 \%$. De este análisis se desprende que en pacientes con bajo riesgo de enfermedad en el tiroides la tiroidectomía podría no ser necesaria.

En presencia de evidencia clínica 0 imagenológica de lesión tiroidea se debe realizar tiroidectomía total. Si la histología tiroidea muestra carcinoma existen 3 escenarios posibles: enfermedad multicéntrica, metástasis de un primario del quiste hacia el tiroides o metástasis del primario tiroideo hacia el quiste ${ }^{5,9}$.

Si se realiza una extrapolación del manejo del cuello en carcinoma papilar en la glándula tiroides, se puede inferir que el vaciamiento ganglionar cervical no mejora el pronóstico, con ganglios clínicamente normales. Sin embargo en el cuello positivo el manejo debe ser con vaciamiento ganglionar cervical seguido de terapia con radioyodo $0^{10,11}$.

Existe poca evidencia de la efectividad de la terapia hormonal luego de una operación de Sistrunk con tiroides in situ. La terapia de sustitución acompañada de medición regular de tiroglobulina en tiroidectomizados puede ser un buen marcador de recurrencia para el seguimiento de esta patología 12,13 .

Cuando se analiza el pronóstico en relación a la administración de terapia con radioyodo, se encuentra que la presencia de esta terapia se asocia a un peor pronóstico; sin embargo tiene el sesgo que los pacientes que han recibido radioyodo normalmente tienen enfermedad avanzada ${ }^{5}$.

Del análisis de la literatura actual pueden razonablemente desprenderse 2 grupos de pacientes de acuerdo a sus características clínicas e histopatológicas:
1. Grupo de bajo riesgo: Constituido por los pacientes jóvenes (menores de 45 años), con tumores menores a $3 \mathrm{~cm}$, sin evidencia de infiltración a tejidos vecinos, sin metástasis y con tiroides clínica e imagenológicamente normal. En este grupo la recomendación podría ser operación de Sistrunk y observación solamente.

2. Grupo de alto riesgo: Mayor de 45 años, tumor mayor a $3 \mathrm{~cm}$, infiltración a tejidos vecinos, antecedentes de radiación cervical, e imagenología con nódulos tiroideos 0 adenopatías cervicales. En este grupo evidentemente el tratamiento debería ser multidisciplinario con operación de Sistrunk, tiroidectomía total, vaciamiento cervical si procede, reemplazo hormonal y terapia con yodo radioactivo ${ }^{14}$.

\section{CONCLUSIÓN}

En 10 años se encontraron 4 casos de CP en QT. Los 4 pacientes consultaron por historia sugerente de QT, sin otros síntomas. El diagnóstico de cáncer generalmente no se sospecha y es un hallazgo en el informe de la biopsia. El manejo inicial es la operación de Sistrunk, en 2 pacientes se asoció tiroidectomía y terapia con radioyodo más terapia con tiroxina, en 1 paciente se indicó terapia de supresión hormonal. Ningún paciente, mientras se mantuvieron en control (Tabla 3), presentó metástasis nodales, ni recidiva hasta el último control del seguimiento.

\section{BIBLIOGRAFÍA}

1. Heshmatı hM, Fatourechi V, Van Heerden JA, et al. Thyroglossal duct carcinoma: report of 12 cases. Mayo Clin Proc 1997; 72: 315-9.

2. WeISS SD, ORLICH CC. Primary papillary carcinoma of a thyroglossal duct cyst: report of a case and literature review. Br J Surg 1991; 78: 87-9.

3. Brentano $H$. Struma aberrata lingual mit druzen metastasen. Deutsch Med Wschr 1911; 37: 665.

4. LiVolsi VA, Perzin KH, Savetsky L. Carcinoma arising in median ectopic thyroid (including thyroglossal duct tissue). Cancer 1974; 34 : 1303-15. 
5. Patel SG, Escrig M, Shaha AR, et al. Management of well differentiated thyroid carcinoma presenting within a thyroglossal duct cyst. $J$ Surg Oncol 2002; 79: 134-9.

6. Yang YJ, Haghir S, Wanamaker JR, et al. Diagnosis of papillary carcinoma in a thyroglossal duct cyst by fine-needle aspiration biopsy. Arch Pathol Lab Med 2000; 124: 139-42.

7. Samara C, Bechrakis I, Kavadias S, et al. Thyroglossal duct carcinoma: case report and review of the literature, with emphasis on CT findings. Neuroradiology 2001; 43: 647-9.

8. Branstetter BF, Weissman JL, Kennedy TL, et al. The CT appearance of thyroglossal duct carcinoma. AJNR Am J Neuroradiol 2000; 21: 1547-50.

9. Martinez-Tello FJ, Martinez-Cabruja R, Fernandez-Matin J, et al. Occult carcinoma of the thyroid. Cancer 1993; 71: 4022-9.

10. Hughes CJ, Shaha AR, Shah JP, et al. Impact of lymph node metastasis in differentiated carci- noma of the thyroid: a matched pair analysis. Head Neck 1996; 18: 127-32.

11. Doshi SV, Cruz RM, Hilsinger RL. Thyroglossal duct carcinoma: a large case series. Ann Otol Rhinol Laryngol 2001; 110: 734-8.

12. Hilger AW, Thompson SD, Smallman LA, et al. Papillary carcinoma arising in thyroglossal duct cyst: a case report and literature review. $J$ Laryngol Otol 1995; 109: 1124-7.

13. SPENCER CA. Thyroglobulin. In The Thyroid. Edited by Braverman LE, Utiger RD. Philadelphia: Lippincott-Raven; 1996; 406-16.

14. Motamed M, McGlashan J. Thyroglossal duct carcinoma. Current Opinion in Otolaryngology \& Head and Neck Surgery 2004; 12: 106-9.

15. Guzmán P, Baeza A. Carcinoma papilar en quiste del conducto tirgloso. Reporte de dos casos. Rev Méd Chile 2008; 136: 1169-74.

16. OJedA JP, Celedón C. Carcinoma papilar del conducto tirogloso. Reporte de dos casos y revisión de la literatura. Rev Otorrinolaringol Cir Cab-Cue 2002; 62: 33-41. 